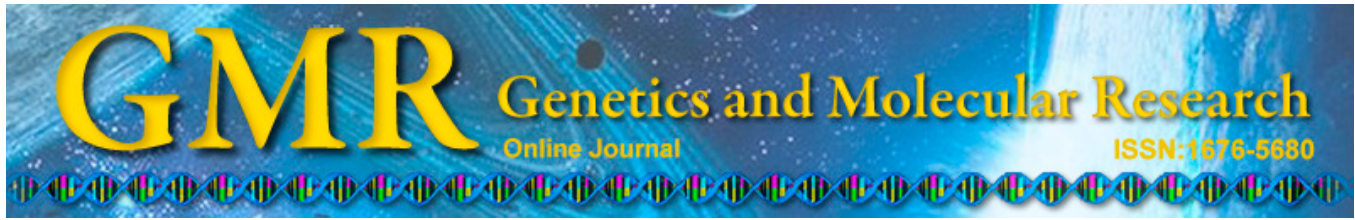

\title{
Identification of smut-responsive genes in sugarcane using cDNA-SRAP
}

\author{
N. Huang ${ }^{1,2 *}$, Y.Y. Zhang ${ }^{1,2 *}$, X.H. Xiao ${ }^{1,2}$, L. Huang ${ }^{1,2}$, Q.B. Wu ${ }^{1,2}$, \\ Y.X. Que ${ }^{1,2}$ and L.P. Xu ${ }^{1,2}$ \\ ${ }^{1}$ Key Laboratory of Sugarcane Biology and Genetic Breeding, \\ Ministry of Agriculture, Fujian Agriculture and Forestry University, \\ Fuzhou, Fujian, China \\ ${ }^{2}$ Sugarcane Research \& Development Center, China Agriculture Research System, \\ Fujian Agriculture and Forestry University, Fuzhou, Fujian, China \\ *These authors contributed equally to this study. \\ Corresponding authors: Y.X. Que / L.P. Xu \\ E-mail: queyouxiong@126.com / xlpmail@126.com
}

Genet. Mol. Res. 14 (2): 6808-6818 (2015)

Received September 15, 2014

Accepted February 11, 2015

Published June 18, 2015

DOI http://dx.doi.org/10.4238/2015.June.18.23

\begin{abstract}
Sugarcane smut, caused by the fungus Sporisorium scitamineum, is one of the main diseases that affect sugarcane worldwide. In the present study, the cDNA-SRAP technique was used to identify genes that are likely to be involved in the response of sugarcane to S. scitamineum infection. In total, 21 bands with significant differential expression during cDNA-SRAP analysis were cloned and sequenced. Real-time qPCR confirmation demonstrated that expression of 19 of these 21 differential bands was consistent with the expression observed during cDNA-SRAP analysis, with a deduced false positive rate of $9.5 \%$. Sequence alignment indicated that 18 of 19 differentially expressed genes showed homologies from 19\% to $100 \%$ to certain genes in GenBank, including the following genes: topoisomerase (EU048780), ethylene insensitive (EU048778), and tetraspanin (EU048770). A real-time qPCR assay showed that during 0-72 $\mathrm{h}$ after pathogen infection, expression of the topoisomerase and
\end{abstract}


the ethylene insensitive genes was upregulated, whereas expression of the tetraspanin gene was downregulated, identical to the expression patterns observed under salicylic acid treatment. Therefore, all three genes are thought to play a role during $S$. scitamineum challenge, but with different functions. To our knowledge, this is the first report on the application of cDNA-SRAP in differential gene expression analysis of sugarcane during a sugarcane-S. scitamineum interaction. The results obtained also contribute to a better understanding of the molecular mechanisms associated with sugarcane-S. scitamineum interactions.

Key words: Sporisorium scitamineum; Differentially expressed gene; Sugarcane; cDNA-SRAP; Real-time qPCR

\section{INTRODUCTION}

Sugarcane is the primary sugar crop in the world and cane sugar accounts for over $75 \%$ of sugar production in the world. During the process of sugarcane cultivation, numerous pathogens infect sugarcane and fungal diseases are the main biotic stress. Sugarcane smut is one of the prevalent fungal diseases of sugarcane and is caused by the fungus Sporisorium scitamineum (Lee-Lovick, 1978; Su et al., 2013a). It can seriously reduce cane and sugar yield and adversely affect juice quality, causing huge economic losses (Hoy et al., 1986). Since the first report of sugarcane smut in 1877 in the Natal region of South Africa, this disease has been a problem in almost all countries in which sugarcane is grown, with the exception of Papua New Guinea until 1990 (Lee-Lovick, 1978; Chao et al., 1990). However, it should be noted that sugarcane smut does not always pose a serious problem because its incidence and severity vary depending on the cultivars grown and the environmental conditions (Que et al., 2012a).

The smut-resistant mechanism of sugarcane is mainly reflected at the morphological, physiological, and molecular levels (Pinon et al., 1999; de Armas et al., 2007). The molecular interaction between sugarcane and $S$. scitamineum has been investigated by different types of molecular techniques, such as cDNA-AFLP (cDNA-amplified fragment length polymorphism) (Thokoane and Rutherford, 2001; Borras-Hidalgo et al., 2005; Lao et al., 2008; Que et al., 2011a), DDRT-PCR (differential-display reverse transcription-PCR) (Que et al., 2009a), cDNA microarray (Que et al., 2009b), TaqMan real-time PCR (Su et al., 2013a), Solexa sequencing (Wu et al., 2013), RNA-Seq (Que et al., 2014a), genome sequencing (Que et al., 2014b), and 2-DE (two-dimensional gel electrophoresis) coupled with MALDI-TOF-TOF/ MS (matrix-assisted laser desorption ionization-time of flight mass spectrometry) (Que et al., 2011b). Compared to the cDNA-AFLP technique, cDNA-SRAP has fewer steps, and the operational procedure is much easier to control ( $\mathrm{Li}$ and Quiros, 2001). Until now, there have been no reports on the application of the cDNA-SRAP technique in differential gene expression of sugarcane under the stress of $S$. scitamineum infection, and the molecular interactions revealed by the cDNA-SRAP method may be helpful in improving understanding of the sugarcane smut-resistance mechanism.

Sequence-related amplified polymorphism (SRAP) is a PCR-based molecular marker technique developed by Li and Quiros (2001) to detect coding sequence polymorphisms. It 
combines simplicity, reproducibility, reasonable throughput ratio, and easy isolation of bands for sequencing. The cDNA-SRAP technique, with cDNA as a template, has been proven to be suitable for analysis of differential gene expression in several plants, including Brassica oleracea (Li et al., 2003; Deng et al., 2007), Spartina angelica (Lu and Wu, 2006), B. napus (Ma et al., 2008) and Erianthus arundinaceum (Que et al., 2012b). Furthermore, the cDNASRAP method, together with agarose gel electrophoresis, can overcome the shortcomings of the isotope labeling and silver staining methods by providing a shorter, safer, and less expensive method that requires no special equipment and can be conducted in a typical laboratory (Que et al., 2012b).

The objective of the present study was to investigate differential gene expression in sugarcane before and after $S$. scitamineum inoculation using a combined method of the cDNASRAP technique and agarose gel electrophoresis. The differential bands obtained from the cDNA-SRAP analysis were then validated using real-time quantitative PCR (qPCR). Finally, the expression profiling of three differentially expressed genes was conducted using real-time qPCR. This study provides experimental evidence for the application of the cDNA-SRAP technique in the differential gene expression analysis of sugarcane during sugarcane-smut interaction and lays the foundation for the isolation of smut-resistant genes from sugarcane, which may have potential applications in sugarcane molecular breeding.

\section{MATERIAL AND METHODS}

\section{Plant material and stress treatments}

The smut-resistant sugarcane variety NCo376 was used in this study and the inoculum source was $S$. scitamineum teliospores, which were collected from sugarcane variety F134. Healthy, vigorous sugarcane plants with uniform growth were selected, and the middle stem was cut into double bud stem segments. Sand cultivation was then conducted in an illumination incubator with a constant temperature of $27^{\circ} \mathrm{C}$, a light intensity of $440 \mathrm{~mol} \cdot \mathrm{m}^{-2} \cdot \mathrm{s}^{-1}, 12 \mathrm{~h}$ light/dark of each day. A $50^{\circ} \mathrm{C}$ hot water treatment for $2 \mathrm{~h}$ was conducted and the detection protocol developed by Albert and Schenck (1996) and Su et al. (2013a) was used to confirm the complete hot water disinfection. All the collected samples were frozen in liquid nitrogen immediately, and stored in a refrigerator at $-80^{\circ} \mathrm{C}$ until RNA extraction.

Stress treatment experiments were conducted on two groups of samples as follows: For group one, inoculation by manual pinpricking was performed at the buds, where three punctures per bud were made with a pin dipped in the teliospore suspension at a concentration of $5 \times 10^{6} \mathrm{~mL}^{-1}$ (Que et al., 2011a, 2009a). For group two, sugarcane plantlets with a height of $15 \mathrm{~cm}$ were transferred into a greenhouse for cultivation in Hoagland nutrient solution. During the six leaves period, evenly growing stalks were selected. After rehydration for $2 \mathrm{~d}$, the stalks were cultivated in Hoagland nutrient solution for one week and $5 \mathrm{mM}$ salicylic acid (SA) was then sprayed onto the leaves (Que et al., 2011a, 2009a). Sampling was conducted at 0, 12, 24, $36,48,60$, and $72 \mathrm{~h}$ after inoculation for group 1 and after salicylic acid application for group 2. For cDNA-SRAP analysis and real-time qPCR confirmation, mRNA from leaves harvested at the six sampling times $(12,24,36,48,60$, and $72 \mathrm{~h})$ was averaged in equal proportions to produce the template cDNA. A sample inoculated with sterile $\mathrm{ddH}_{2} \mathrm{O}$ was used as the negative control. 


\section{RNA extraction and cDNA synthesis}

Total RNA from sugarcane leaves was extracted according to the protocol described by Que et al. (2008). The quantity and quality of extracted RNA was determined using agarose gel electrophoresis and a UV spectrophotometer. RNA was treated with RNase-free DNase I (Amersham Pharmacia Biotech Co., Ltd., Tokyo, Japan) at a ratio of $1 \mathrm{U}$ DNase I to $20 \mu \mathrm{g}$ RNA and incubated for $20 \mathrm{~min}$ at $37^{\circ} \mathrm{C}$. First strand cDNA from $10 \mu \mathrm{g}$ DNase-I-treated RNA and second strand cDNA were generated using a SMART ${ }^{\mathrm{TM}}$ cDNA synthesis kit (Clontech Laboratories, Inc., CA, USA).

\section{cDNA-SRAP analysis}

The cDNA-SRAP procedure described by Que et al. (2012b) was utilized with a minor modification and the primer sequences were selected from previous reports (Table 1) (Li et al., 2003; Deng et al., 2007; Que et al., 2012b). The $50 \mu \mathrm{L}$ cDNA-SRAP reaction contained $5.0 \mu \mathrm{L}$ 10X PCR buffer ( $15 \mathrm{mM} \mathrm{MgCl}_{2}$ plus), $3.75 \mu \mathrm{L}$ dNTPs $(10 \mathrm{mM}), 1.25 \mathrm{U}$ Taq DNA polymerase enzyme, $1.5 \mu \mathrm{L}$ forward and reverse primers $(10 \mu \mathrm{M})$ respectively, and $100 \mathrm{ng}$ second strand cDNA as the template. PCR cycling conditions comprised an initial cycle at $94^{\circ} \mathrm{C}$ for 5 min, five cycles at $94^{\circ} \mathrm{C}$ for $1 \mathrm{~min}, 35^{\circ} \mathrm{C}$ for $1 \mathrm{~min}$, and $72^{\circ} \mathrm{C}$ for $1 \mathrm{~min}$, followed by 35 cycles at $94^{\circ} \mathrm{C}$ for $1 \mathrm{~min}, 50^{\circ} \mathrm{C}$ for $1 \mathrm{~min}$, and $72^{\circ} \mathrm{C}$ for $1 \mathrm{~min}$, and a final extension at $72^{\circ} \mathrm{C}$ for 10 min. The primers used in cDNA-SRAP analysis are shown in Table 2.

The cDNA-SRAP amplified products were concentrated to approximately $10 \mu \mathrm{L}$ using a centrifugal freeze drying system (Martin Christ, Germany) (Que et al., 2012b). The total volume was loaded onto a $1.6 \%$ agarose gel (containing $0.1 \%$ Ethidium bromide) for electrophoresis and imaged with a Bio-Rad Gel Imaging System (Hercules, CA, USA). The Agarose SFRTM was purchased from Sangon Biological Engineering Technology and Services Co., Ltd, Shanghai, China.

\section{Function annotation of differentially expressed genes}

The differentially expressed genes obtained were subjected to a BlastX similarity search analysis against the NCBI protein database and annotated according to their homologies with known protein sequences (Que et al., 2012b; Su et al., 2014a).

\section{Real-time qPCR confirmation and expression profile analysis of differentially expressed genes}

Real-time qPCR confirmation was performed for all of the 21 differential bands screened in the cDNA-SRAP analysis. Three differentially expressed genes of interest, topoisomerase gene (EU048780), ethylene insensitive gene (EU048778), and tetraspanin gene (EU048770), were selected for expression profile analysis. Primers specific to the sugarcane 25S rRNA gene were used for normalization of the reactions (Ling et al., 2014), and real-time qPCR primers were designed using Primer premier 5.0 (Table 2).

Real-time qPCR was performed in an ABI PRISM 7500 Real-Time PCR System (Life Technologies, CA, USA). The amplification reaction contained $2.5 \mu \mathrm{L}(40 \mathrm{ng} / \mu \mathrm{L})$ second strand cDNA template, $12.5 \mu \mathrm{L}$ SYBR Primix ExTaq $(2 \mathrm{X}), 0.5 \mu \mathrm{L}$ each forward and reverse 
primers $(10 \mu \mathrm{M})$, and $9.0 \mu \mathrm{L}$ sterile water to a final volume of $25 \mu \mathrm{L}$. Each sample was replicated three times. The following thermal cycling profile was used: $5^{\circ} \mathrm{C}$ for $30 \mathrm{~s} ; 40$ cycles of $95^{\circ} \mathrm{C}$ for $5 \mathrm{~s}$, and $60^{\circ} \mathrm{C}$ for $34 \mathrm{~s}$. When the reaction finished, the melting curve was analyzed. Fold change in gene expression was estimated using threshold cycles and the $2^{-\Delta \Delta \mathrm{CT}}$ method (Livak and Schmittgen, 2001; Pfaffl, 2001; Su et al., 2013b, 2014b).

\section{RESULTS}

\section{cDNA-SRAP analysis}

cDNA-SRAP fingerprints of sugarcane before and after inoculation with S. scitamineum were developed using 50 primer combinations. Of these 50 combinations, seven generated 28 different bands from a total of 170 bands (data not shown). In order to ensure reliability of differential expression, only 21 bands with significant differential expression were cloned and sequenced. These 21 bands were cDNA-SRAP markers assumed to respond to inoculation by $S$. scitamineum. The band sequences and their corresponding primer combinations are illustrated in Table 1. Among these bands, 12 were upregulated and nine were downregulated (Table 3).

\begin{tabular}{|c|c|c|c|}
\hline No. & Primer codes and sequences $\left(5^{\prime}-3^{\prime}\right)$ & No. & Primer codes and sequences $\left(5^{\prime}-3^{\prime}\right)$ \\
\hline 1 & mel: 5'-TGAGTCCAAACCGGATA-3' & 9 & em4: 5'-GACTGCGTACGAATTTGA-3' \\
\hline 2 & me2: 5'-TGAGTCCAAACCGGAGC-3' & 10 & ern5: 5'-GACTGCGTACGAATTAAC-3' \\
\hline 3 & me3: 5'-TGAGTCCAAACCGGAAT-3' & 11 & em6: 5'-GACTGCGTACGAATTGCA-3' \\
\hline 4 & me4: 5'-TGAGTCCAAACCGGACC-3' & 12 & em7: 5'-GACTGCGTACGAATTATG-3' \\
\hline 5 & me5: 5'-TGAGTCCAAACCGGAAG-3' & 13 & em12: 5'-GACTGCGTACGAATTGTC-3' \\
\hline 6 & em1: 5'-GACTGCGTACGAATTAAT-3' & 14 & em13: 5'-GACTGCGTACGAATTGGT-3' \\
\hline 7 & em2: 5'-GACTGCGTACGAATTTGC-3' & 15 & em15: 5'-GACTGCGTACGAATTCTG-3' \\
\hline 8 & em3: 5'-GACTGCGTACGAATTGAC-3' & & \\
\hline
\end{tabular}

\section{Real-time qPCR confirmation of 21 differential bands screened in cDNA-SRAP analysis}

The primers were designed based on the sequences of 21 different bands identified in the cDNA-SRAP analysis (Table 2), and cDNA from sugarcane before and after the stress of $S$. scitamineum infection was selected as the template (the same as that used in the cDNA-SRAP analysis). The results demonstrate that the expression of 19 of 21 different bands by real-time qPCR is consistent with the expression observed using cDNA-SRAP analysis, with the exception of EU048777 and EU048787 (Table 3). The deduced false positive rate was (21-19)/21 $=9.5 \%$.

\section{Gene function annotation of 19 differentially expressed genes screened in cDNA- SRAP analysis}

Sequence alignment indicated that 18 of the 19 differentially expressed genes showed homologies from 19 to $100 \%$ to certain genes in GenBank, while the remaining gene (EU048790) showed no significant homology with reported genes (Table 3). 
Table 2. Sequences of primers used in real-time qPCR confirmation of differentially expressed genes from the cDNA-SRAP analysis.

\begin{tabular}{clll}
\hline No. & Primer code & Forward primer sequence (5'-3') & Reverse primer sequence (5'-3') \\
\hline 1 & EU048767 & 5'-AAACTGCGTCCAAGCCATCT-3' & 5'-TTCTTCTGACCCCGCCTTT-3' \\
2 & EU048768 & 5'-TCTGGACACGTTGATATGCTTTG-3' & 5'-GAAGTGCTGTGAAGAGTGAGGTGT-3' \\
3 & EU048769 & 5'-GGGTAGTCGACACCACTGAG-3' & 5'-CCAAGGGCAATAAACAGGAG-3' \\
4 & EU048770 & 5'-CATGGAGCCAAAGAATGAGA-3' & 5'-TCAGAGAATTGTGCATCGGT-3' \\
5 & EU048771 & 5'-CGGACTGATCGGGACTCTAT-3' & 5'-TCTCCATTTGGTGCATTGAT-3' \\
6 & EU048772 & 5'-AAACCGGACCACGGAAGA-3' & 5'-ATTCCGCACCCCGTTAGA-3' \\
7 & EU048773 & 5'-AGATAAAAGGGCAGGGGAGAA-3' & 5'-GCTTGTTTTCGGTGATGGTG-3' \\
8 & EU048774 & 5'-CAAGAACTGCAAGTGTGTTGAA-3' & 5'-GAGATTCAGTATCATAACGGTTTCC-3' \\
9 & EU048775 & 5'-AGGCCATTTCAACTCTTGCTCTAC-3' & 5'-TGACGGGACACTGAGGACTTT-3' \\
10 & EU048776 & 5'-ATAGGGCGATTGTCACTGCTT-3' & 5'-TCGGACCTCTGCTTAGTTTCATC-3' \\
11 & EU048777 & 5'-GGCACAACTCCCACCAGAA-3' & 5'-GGAACTATCAGCAAGCCACCA-3' \\
12 & EU048778 & 5'-CAACACTTTCTACTGTGCTTTCCTC-3' & 5'-CCCCTCCCCGTCATTTACTT-3' \\
13 & EU048779 & 5'-CTGCCATTGGAGGGTCATCT-3' & 5'-TTGAGGTTGATGAGGAGTTGCTT-3' \\
14 & EU048780 & 5'-TCACGTCTCGACACCACCA-3' & 5'-GGAACCCCAAAAGCGAAAA-3' \\
15 & EU048782 & 5'-TGAACCTCTCTTGGATTCCC-3' & 5'-TGGGAAGTCATAGCCAACAA-3' \\
16 & EU048783 & 5'-GATTGCACCAATAACGCATC-3' & 5'-AAACAAGCGGCCAATATTTC-3' \\
17 & EU048784 & 5'-AACTCATTGCATTTGGGACA-3' & 5'-ATGTTGTTACGCCGTTCCTT-3' \\
18 & EU048785 & 5'-TGGATCACCGTTCTCATTTCTCT-3' & 5'-CTAAGATGGTGTGCTGTGCTATCC-3' \\
19 & EU048786 & 5'-GAGTCCAGCATATCCTTCCGTTTA-3' & 5'-TGCCAAGTAGTCCAGGTTTTCC-3' \\
20 & EU048787 & 5'-GGCACAACTCCCACCAGAA-3' & 5'-GGAACTATCAGCAAGCCACCA-3' \\
21 & EU048790 & 5'-CCCTCCTGGAGTTTACTTGC-3' & 5'-CATGTGAATTTGAGAGGCAGA-3' \\
\hline
\end{tabular}

Table 3. Differentially expressed genes during sugarcane-smut interaction identified using cDNA-SRAP analysis and their homologies to genes in GenBank.

\begin{tabular}{|c|c|c|c|c|c|}
\hline No. & Accession No. & $\begin{array}{l}\text { Primer } \\
\text { combination }\end{array}$ & Homology with BlastX (Accession No.) & $\begin{array}{c}\text { Similarity } \\
(\%)\end{array}$ & E-value \\
\hline 1 & EU048767* & $\mathrm{Me} 2 / \mathrm{Em} 13$ & sec14p-like phosphatidylinositol transfer family protein [Zea mays] (AFW70029.1) & 99 & $6 \mathrm{e}^{-78}$ \\
\hline 2 & EU048768 & Me1/Em6 & Negative regulator of mitosis [Sporisorium reilianum] (CBQ71170.1) & 19 & $2 \mathrm{e}^{-07}$ \\
\hline 3 & EU048769* & $\mathrm{Me} 3 / \mathrm{Em} 6$ & Hypothetical protein [Zea mays] (ACG36409.1) & 100 & $3 \mathrm{e}^{-112}$ \\
\hline 4 & EU048770 & Me3/Em6 & Tetraspanin family protein [Zea mays] (NP_001150827.1) & 67 & $2 \mathrm{e}^{-07}$ \\
\hline 5 & EU048771 & $\mathrm{Me} 3 / \mathrm{Em} 6$ & PHD finger-like protein [Oryza sativa Japonica Group] (NP_001046163.1) & 99 & $2 \mathrm{e}^{-103}$ \\
\hline 6 & EU048772* & $\mathrm{Me} 4 / \mathrm{Em} 5$ & acyl-CoA synthetase [Aeromicrobium marinum DSM 15272] (ZP_07715729.1) & 74 & 3.1 \\
\hline 7 & EU048773 & $\mathrm{Me} 4 / \mathrm{Em} 5$ & transcription-repair coupling factor [Actinobacillus succinogenes] $\mathrm{YP}$ _001343867.1) & 33 & 0.43 \\
\hline 8 & EU048774 & $\mathrm{Me} 4 / \mathrm{Em} 5$ & Ribosomal protein L22 (AA 1-126) [Zea mays] (CAA68422.1) & 29 & $5 \mathrm{e}^{-05}$ \\
\hline 9 & EU048775 & Ie5/Em15 & Lon protease homolog 2, peroxisomal [Zea mays] (XP_002462726.1) & 48 & $6 \mathrm{e}^{-114}$ \\
\hline 10 & EU048776* & $\mathrm{Me} 2 / \mathrm{Em} 13$ & Cell wall-associated hydrolase, partial [Medicago truncatula] (XP_003637074.1) & 51 & $7 \mathrm{e}^{-21}$ \\
\hline 11 & EU048777* & $\mathrm{Me} 3 / \mathrm{Em} 3$ & Hypothetical protein SORBIDRAFT [Sorghum bicolor] (XP_002452600.1) & 99 & $4 \mathrm{e}^{-141}$ \\
\hline 12 & EU048778* & $\mathrm{Me} 1 / \mathrm{Em} 4$ & Ethylene insensitive 2 [Zea mays] (AAR25570.1) & 99 & $9 \mathrm{e}^{-54}$ \\
\hline 13 & EU048779 & $\mathrm{Me} 1 / \mathrm{Em} 4$ & Transposon protein [Oryza sativa Japonica Group] (ABA91313.1) & 93 & $2 \mathrm{e}^{-67}$ \\
\hline 14 & EU048780* & $\mathrm{Me} 3 / \mathrm{Em} 6$ & DNA topoisomerase $2[$ Triticum urartu $]$ (EMS63570.1) & 72 & $4 e^{-10}$ \\
\hline 15 & EU048782 & $\mathrm{Me} 4 / \mathrm{Em} 2$ & Alpha-glucanphosphorylase, hisozyme [Oryza sativa Indica Group] (ABR26152.1) & 97 & $1 \mathrm{e}^{-41}$ \\
\hline 16 & EU048783* & $\mathrm{Me} 4 / \mathrm{Em} 2$ & Glyoxalase [Streptomyces violaceusniger] (YP_004816136.1) & 59 & 4.5 \\
\hline 17 & EU048784* & $\mathrm{Me} 4 / \mathrm{Em} 2$ & Lysine exporter protein LysE [Burkholderia sp. SJ98] (ZP 11401116.1) & 45 & 4.2 \\
\hline 18 & EU048785* & $\mathrm{Me} 4 / \mathrm{Em} 15$ & Cell division control protein 2 homolog 2 [Sorghum bicolor] (CAZ96037.1) & 100 & $6 \mathrm{e}^{-64}$ \\
\hline 19 & EU048786* & $\mathrm{Me} 5 / \mathrm{Em} 15$ & Hypothetical protein [Sorghum bicolor] (XP_002459683.1) & 91 & $1 \mathrm{e}^{-57}$ \\
\hline 20 & EU048787 & $\mathrm{Me} 3 / \mathrm{Em} 3$ & Hypothetical protein [Sorghum bicolor] (XP_002452600.1) & 99 & $1 \mathrm{e}^{-141}$ \\
\hline 21 & EU048790* & $\mathrm{Me} 4 / \mathrm{Em} 5$ & No significant similarity found & & \\
\hline
\end{tabular}

*Denotes differentially expressed genes upregulated in sugarcane infected by Sporisorium scitamineum, and the remaining genes downregulated. EU048777 and EU048787 are the two genes with different expression patterns between cDNA-SRAP analysis and real-time qPCR confirmation.

\section{Expression profile analysis of three differentially expressed genes by real-time qPCR}

A real-time qPCR assay was used to validate the expressions of three differentially expressed genes in sugarcane infected with S. scitamineum (Figures 1, 2, and 3). The smut- 
resistant genotype NCo376 was selected as the plant material. The results showed that during 0-72 $\mathrm{h}$ after pathogen infection, the topoisomerase and the ethylene insensitive genes were upregulated, whereas the tetraspanin gene was downregulated, the same responses as those observed under SA treatment. Therefore, all three differentially expressed genes may play a role during S. scitamineum infection; however, with different functions. This also suggests that the smut-resistance function mechanism differs among these three genes.

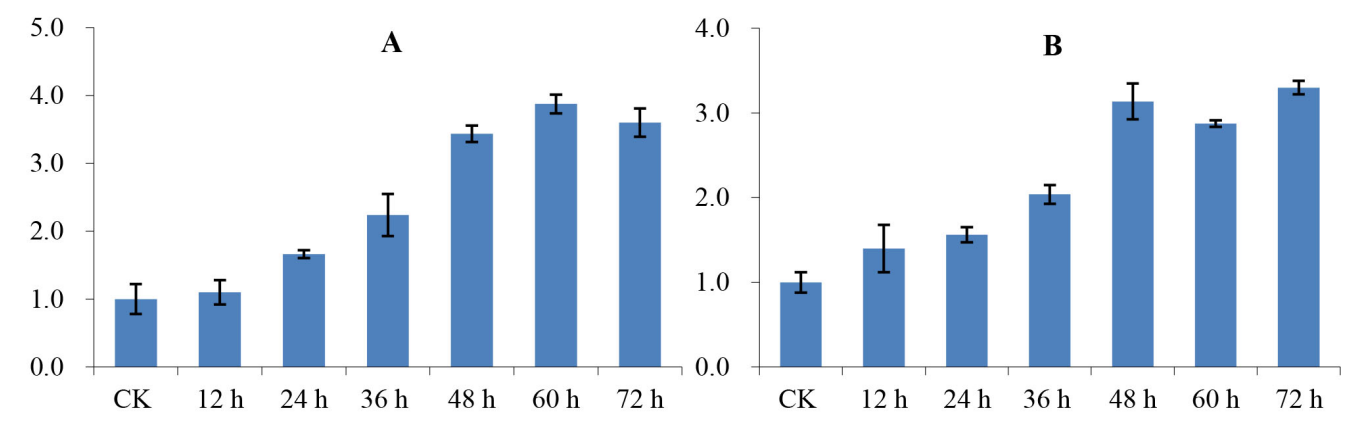

Figure 1. Expression profile analysis of the topoisomerase gene (EU048780) in sugarcane under salicylic acid (A) and Sporisorium scitamineum $(\mathbf{B})$ treatments using real-time qPCR. X-axis = treatment time; $y$-axis $=$ relative expression.

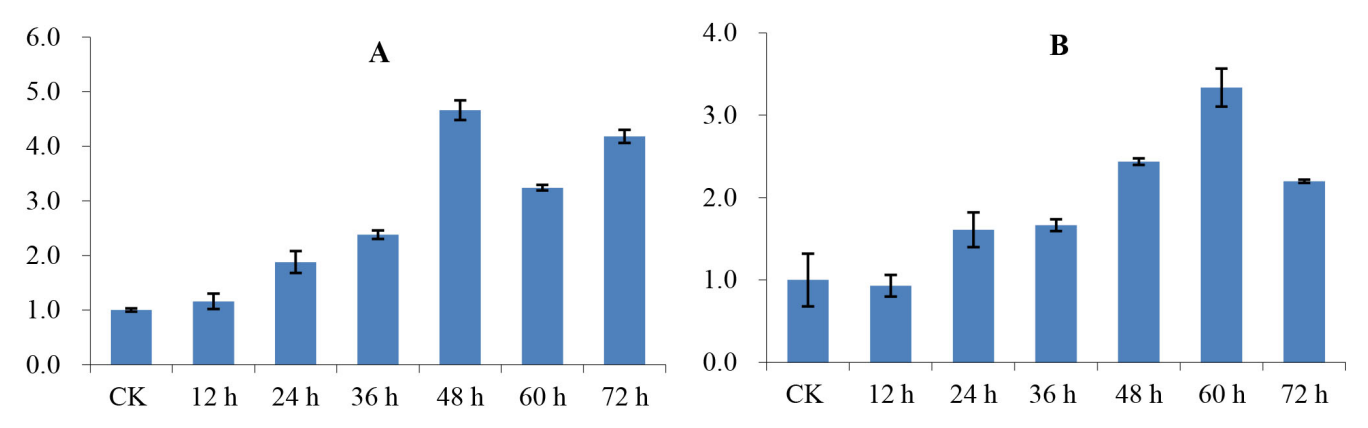

Figure 2. Expression profile analysis of the ethylene insensitive gene (EU048778) in sugarcane under salicylic (A) and Sporisorium scitamineum $(\mathbf{B})$ treatments using real-time $\mathrm{qPCR}$. X-axis $=$ treatment time; $\mathrm{y}$-axis $=$ relative expression.
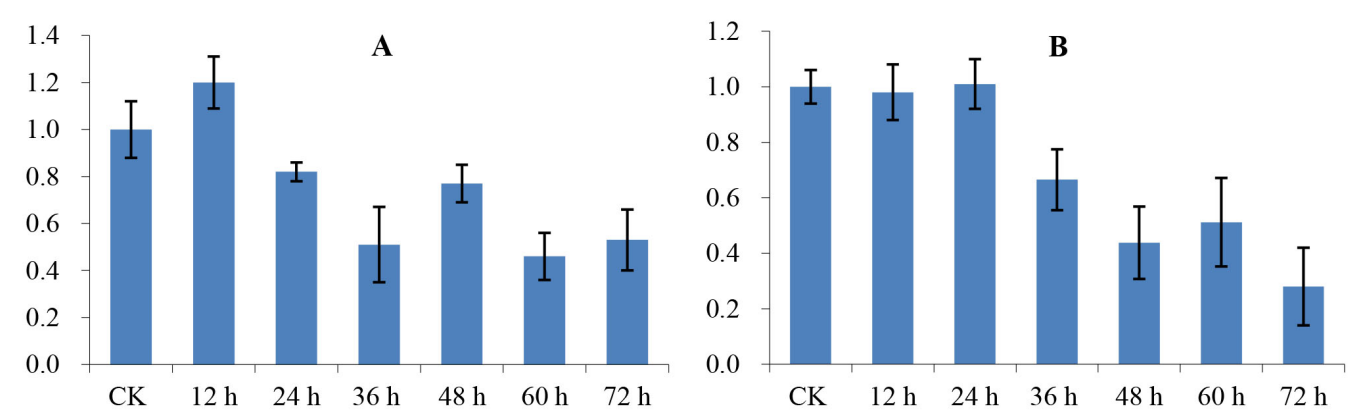

Figure 3. Expression profile analysis of the tetraspanin gene (EU048770) in sugarcane under salicylic acid (A) and Sporisorium scitamineum (B) treatments using real-time qPCR. $\mathrm{X}$-axis $=$ treatment time; $\mathrm{y}$-axis $=$ relative expression. 


\section{DISCUSSION}

Plant disease resistance is the result of mutual adaptation and selection between the plant and pathogen during long-term co-evolution (Graham and Graham, 1991). A series of physiological and biochemical changes, along with molecular responses, are assumed to occur during the period from the beginning of the $S$. scitamineum stress on sugarcane due to infection by the pathogen to the interaction between sugarcane and $S$. scitamineum (Pinon et al., 1999; Thokoane and Rutherford, 2001; Borras-Hidalgo et al., 2005; de Armas et al., 2007; Lao et al., 2008; Que et al., 2009a,b, 2011a,b, 2014a,b; Su et al., 2013a; Wu et al., 2013). In the present study, the polymorphic bands of the cDNA-SRAP gel showed abundant alterations among sugarcane before and after the stress of $S$. scitamineum infection, suggesting that cDNA-SRAP could become an effective technique to globally compare differential gene expression in sugarcane during sugarcane-smut interactions.

Previous research has revealed that cDNA-SRAP is a simple, reproducible, and reasonable-throughput approach for the comprehensive analysis of differential gene expression at the cDNA level (Li et al., 2003; Lu and Wu, 2006; Deng et al., 2007; Ma et al., 2008; Que et al., 2012b). In the present study, cDNA-SRAP along with agarose gel electrophoresis, was adopted to analyze how genes in sugarcane function during sugarcane-S. scitamineum interactions. In total, 21 differential bands, 12 upregulated and 9 downregulated, were identified, showing the high-throughput efficiency of the cDNA-SRAP technique. Through realtime qPCR confirmation, the expression of 19 of these 21 genes was found to be consistent with that observed using cDNA-SRAP analysis, and the remaining two were false positive, accounting for only $9.5 \%$. This rate, when compared with a false positive rate of almost $70 \%$ in DDRT-PCR, is a distinct advantage (Que et al., 2009a); however, the rate is slightly higher than the $6.5 \%$ observed in cDNA-SRAP analysis of E. arundinaceum (Que et al., 2012b). From this point of view, the cDNA-SRAP technique is a feasible and relatively stable technique that can be employed in the study of differential gene expression in sugarcane during the sugarcane-smut interaction.

Identification of differentially expressed genes under certain circumstances is likely to provide clues as to what types of defense mechanisms and biochemical pathways are regulated under this type of stress (Sävenstrand et al., 2000). In the present study, all of the 21 differential bands obtained in the cDNA-SRAP analysis were selected for sub-cloning and sequencing, of which 19 were further validated to be truly differentially expressed genes using real-time qPCR. Bioinformatic analysis indicated that all of the 19 differentially expressed genes obtained in this study may play a role in the interaction response of sugarcane to the $S$. scitamineum challenge, but with different functions. The differentially expressed genes are involved in several different metabolic pathways, such as transcription repair, cell division, and acyl-CoA synthetase. It should also be noted that, although the sequence alignment of 19 differentially expressed sugarcane genes that emerged from the cDNA-SRAP analysis do not appear to be directly linked with the stress of $S$. scitamineum because of their differential expression before and after the $S$. scitamineum infection and the complex nature of the smut resistance mechanism, they could be smut-responsive genes in sugarcane. Based on the above results, a complicated gene and protein regulatory network is assumed to be involved in the interaction of the sugarcane-S. scitamineum biosystem.

The signal factor SA is a monohydroxybenzoic acid, a type of phenolic acid and a beta hydroxy acid. It has been proven to play an important role in signal transduction and the 
resistance response of plants during the early stages of infection, such as the hypersensitive response and systemic acquired resistance (Durner et al., 1997). Topoisomerases are isomerase enzymes that act on the topology of DNA and regulate the overwinding or underwinding of DNA (Champoux, 2001). The ethylene insensitive gene, a bifunctional transducer of ethylene and stress responses, is a central component of the ethylene signaling pathway (Alonso et al., 1999). This gene has been shown to be involved in the activation of ethylene responses and the restoration of responsiveness to abiotic and biotic stresses, including abscisic acid and jasmonic acid treatment, ozone stress, high salt stress, oxidative stress, and disease resistance (Cao et al., 2006; Wang et al., 2007; Que et al., 2012b). Tetraspanins, also called tetraspans or the transmembrane 4 superfamily (TM4SF), are a family of membrane proteins found in all multicellular eukaryotes (Hemler, 2005). They are often thought to act as scaffolding proteins, anchoring multiple proteins to one area of the cell membrane (Hemler, 2005). In this study, under the stress of $S$. scitamineum, the expression profiles of three differentially expressed genes (the topoisomerase gene, the ethylene insensitive gene, and the tetraspanin gene) were the same as those observed under SA treatment. This further confirms that these three genes could be smut-responsive genes during sugarcane-S. scitamineum interactions. The upregulation of the topoisomerase (EU048780) and the ethylene insensitive (EU048778) genes may indicate their positive responses to smut stress, while the downregulation of the tetraspanin gene (EU048770) indicates its negative response to smut stress. These three genes are assumed to be involved in both plant defense responses and sugarcane smut resistance, and further research is needed to determine their usefulness in breeding new smut-resistant sugarcane cultivars.

For the first time, the present study reports differential gene expression in sugarcane during the sugarcane-S. scitamineum interaction using cDNA-SRAP along with agarose gel electrophoresis. These results also provide some molecular biological evidence for the mechanisms associated with the sugarcane-S. scitamineum interaction.

\section{ACKNOWLEDGMENTS}

Research funded by the Natural Science Foundation of Fujian Province, China (\#2015J06006), the National Natural Science Foundation of China (\#31101196), the Program for New Century Excellent Talents in Fujian Province University (\#JA14095), the Research Funds for Distinguished Young Scientists in Fujian Provincial Department of Education (\#JA13090), and the earmarked fund for China Agriculture Research System (\#CARS-20).

\section{REFERENCES}

Albert HH and Schenck S (1996). PCR amplification from a homolog of the $b E$ mating-type gene as a sensitive assay for the presence of Ustilago scitaminea DNA. Plant Dis. 80: 1189-1192.

Alonso JM, Hirayama T, Roman G, Nourizadeh S, et al. (1999). EIN2, a bifunctional transducer of ethylene and stress responses in Arabidopsis. Science 284: 2148-2152.

Borras-Hidalgo O, Thomma BP, Carmona E, Borroto CJ, et al. (2005). Identification of sugarcane genes induced in disease-resistant somaclones upon inoculation with Ustilago scitaminea or Bipolaris sacchari. Plant Physiol. Biochem. 43: 1115-1121.

Cao SQ, Jiang ST and Zhang RX (2006). Evidence for a role of ethylene-insensitive 2 gene in the regulation of the oxidative stress response in Arabidopsis. Acta Physiol. Plant 28: 417-425.

Champoux JJ (2001). DNA topoisomerases: structure, function, and mechanism. Annu. Rev. Biochem. 70: 369-413. 
Chao CP, Hoy JW, Saxton AM and Martin FA (1990). Heritability of resistance and repeatability of clone reactions to sugarcane smut in Louisiana. Phytopathology 80: 622-627.

de Armas R, Santiago R, Legaz ME, Vicente C (2007). Levels of phenolic compounds and enzyme activity can be used to screen for resistance of sugarcane to smut (Ustilago scitaminea). Austral. Plant Pathol. 36: 32-38.

Deng XH, Zhang SN and Hou XL (2007). Differential expression analysis of bud of pol CMS and its maintainter line of Brassica rapa. ssp. chinensis through SRAP. Acta Hortic. Sin. 34: 655-658.

Durner J, Shah J and Klessig DF (1997). Salicylic acid and disease resistance in plants. Trends Plant Sci. 2: 266-274.

Graham TL and Graham MY (1991). Cellular coordination of molecular responses in plant defense. Mol. Plant-Microbe Interact. 4: 415-422.

Hemler ME (2005). Tetraspanin functions and associated microdomains. Nat. Rev. Mol. Cell Biol. 6: 801-811.

Hoy JW, Hollier CA, Fontenot DB and Grelen LB (1986). Incidence of sugarcane smut in Louisiana and its effects on yield. Plant Dis. 70: 59-60.

Lao M, Arencibia AD, Carmona ER, Acevedo R, et al. (2008). Differential expression analysis by cDNA-AFLP of Saccharum spp. after inoculation with the host pathogen Sporisorium scitamineum. Plant Cell Rep. 27: 1103-1111.

Lee-Lovick G (1978). Smut of sugarcane-Ustilago scitaminea. Rev. Plant Pathol. 147: 181-188.

Li G and Quiros CF (2001). Sequence-related amplified polymorphism (SRAP), a new marker system based on a simple PCR reaction: its application to mapping and gene tagging in Brassica. Theor. Appl. Genet. 103: 455-461.

Li G, Gao M, Yang B and Quiros CF (2003). Gene for gene alignment between the Brassica and Arabidopsis genomes by direct transcriptome mapping. Theor. Appl. Genet. 107: 168-180.

Ling H, Wu QB, Guo J, Xu L, et al. (2014). Comprehensive selection of reference genes for gene expression normalization in sugarcane by real time quantitative RT-PCR. PLoS One 9: e97469.

Livak KJ and Schmittgen TD (2001). Analysis of relative gene expression data using real-time quantitative PCR and the $2^{-\Delta \Delta \mathrm{Ct}}$ method. Methods 25: 402-408.

Lu YQ and Wu WR (2006). Identification of salt-responsive genes in English cordgrass Spartina anglica roots using SRAP technique. J. Zhejiang Univ. 32: 511-514.

Ma AF, Li JN, Chen L, Qian W, et al. (2008). Differential display of related genes to seed-coat color by cDNA-SRAP in Brassica napus L. Acta Agron. Sin. 34: 526-529.

Pfaffl MW (2001). A new mathematical model for relative quantification in real-time RT-PCR. Nucleic Acids Res. 29: 2002-2007.

Pinon D, de Armas R, Vicente C and Legaz ME (1999). Role of polyamines in the infection of sugarcane buds by Ustilago scitaminea spores. Plant Physiol. Biochem. 37: 57-64.

Que YX, Li W, Xu JS, Xu LP, et al. (2008). A simple and versatile protocol for isolation of RNA from plant, fungi and animal. J. Agric. Sci. Technol. 2: 63-63.

Que YX, Yang ZX, Xu LP and Chen RK (2009a). Isolation and identification of differentially expressed genes in sugarcane infected by Ustilago scitaminea. Acta Agron. Sin. 35: 452-458.

Que YX, Xu LP, Lin JW, Xu JS, et al. (2009b). Application of E. arundinaceus cDNA microarray in the study of differentially expressed genes induced by U. scitaminea. Acta Agron. Sin. 35: 940-945.

Que YX, Lin JW, Song XX, Xu LP, et al. (2011a). Differential gene expression in sugarcane in response to challenge by fungal pathogen Ustilago scitaminea revealed by cDNA-AFLP. J. Biomed. Biotechnol. 2011: Article ID 160934.

Que YX, Xu LP, Lin JW, Ruan MH, et al. (2011b). Differential protein expression in sugarcane during sugarcaneSporisorium scitamineum interaction revealed by 2-DE and MALDI-TOF-TOF/MS. Compar. Funct. Genom. 2011: Article ID 989016.

Que YX, Xu LP, Lin JW, Chen RK, et al. (2012a). Molecular variation of Sporisorium scitamineum in mainland China revealed by RAPD and SRAP markers. Plant Dis. 96: 1519-1525.

Que YX, Xu LP, Lin JW, Luo J, et al. (2012b). cDNA-SRAP and its application in differential gene expression analysis: A case study in Erianthus arundinaceum. J. Biomed. Biotechnol. 2012: Article ID 390107.

Que YX, Su YC, Guo JL, Wu QB, et al. (2014a). A global view of transcriptome dynamics during Sporisorium scitamineum challenge in sugarcane by RNA-seq. PLoS One 9: e106476.

Que YX, Xu LP, Wu QB, Liu YF, et al. (2014b). Genome sequencing of Sporisorium scitamineum provides insights into the pathogenic mechanisms of sugarcane smut. BMC Genomics 15: 996.

Sävenstrand H, Brosché M, Ängehagen M and Strid A (2000). Molecular markers for ozone stress isolated by suppression subtractive hybridization: specificity of gene expression and identification of a novel stress-regulated gene. Plant Cell Environ. 23: 689-700.

Su YC, Wang SS, Guo JL, Xue BT, et al. (2013a). A TaqMan real-time PCR assay for detection and quantification of Sporisorium scitamineum in sugarcane. Sci. World J. 2013: Article ID 942682. 
Su YC, Xu LP, Xue BT, Wu QB, et al. (2013b). Molecular cloning and characterization of two pathogenesis-related $\beta-1,3-$ glucanase genes $S c G l u A 1$ and $S c G l u D 1$ from sugarcane infected by Sporisorium scitamineum. Plant Cell Rep. 32: 1503-1519.

Su YC, Xu LP, Fu ZW, Yang YT, et al. (2014a). ScChi, encoding an acidic class III chitinase of sugarcane, confers positive responses to biotic and abiotic stresses in sugarcane. Int. J. Mol. Sci. 15: 2738-2760.

Su YC, Guo JL, Ling H, Chen SS, et al. (2014b). Isolation of a novel peroxisomal catalase gene from sugarcane, which is responsive to biotic and abiotic stresses. PLoS One 9: e84426.

Thokoane LN and Rutherford RS (2001). cDNA-AFLP differential display of sugarcane (Saccharum spp, hybrids) genes induced by challenge with the fungal pathogen Ustilago scitaminea (sugarcane smut). Proc. S. Afr. Sug. Technol. Ass. 75: 104-107.

Wang Y, Liu C, Li K, Sun F, et al. (2007). Arabidopsis EIN2 modulates stress response through abscisic acid response pathway. Plant Mol. Biol. 64: 633-644.

Wu QB, Xu LP, Guo JL, Su YC, et al. (2013). Transcriptome profile analysis of sugarcane responses to Sporisorium scitaminea infection using Solexa sequencing technology. BioMed Res. Int. 2013: Article ID 298920. 